\title{
Juicios multilingües en Barcelona desde la perspectiva de la sociología del lenguaje: lenguas dominantes, lenguas minorizadas y lenguas invisibles
}

Multilingual courts in Barcelona from the perspective of the sociology of language: Dominant, minoritized and invisible languages

\section{Mireia Vargas-Urpi \\ Universitat Autònoma de Barcelona España}




\section{Resumen}

Catalunya es conocida por ser una región bilingüe con políticas lingüísticas que apoyan y promueven el uso del catalán. Paralelamente, en los últimos años, el número de lenguas habladas en Catalunya ha aumentado de manera considerable debido a la llegada de inmigrantes y turistas. En este contexto, la interpretación judicial se ha convertido en un elemento imprescindible cuando personas que no hablan las lenguas oficiales se ven involucradas en un juicio, ya sea como acusados o testigos. En este artículo nos centramos en dos cuestiones que podrían aportar una nueva perspectiva al estudio del multilingüismo en el ámbito judicial. Basándonos en datos extraídos de un corpus de grabaciones de 55 juicios celebrados en Barcelona en los que hubo interpretación, analizamos la diglosia en el ámbito judicial en dos niveles: por un lado, en el uso desigual de las dos lenguas cooficiales en Catalunya (catalán y castellano) y, por el otro, en el uso de lenguas francas (como el inglés o el francés) para acusados o testigos con otras lenguas maternas. Los resultados reflejan que el castellano es claramente la lengua dominante en los juicios y que, efectivamente, el inglés y el francés se utilizan como lenguas francas en juicios con interpretación, a pesar de los distintos niveles de competencia que puedan tener los usuarios de estas lenguas.

Palabras clave: interpretación judicial; juicios multilingües; diglosia; barreras lingüísticas.

\section{Abstract}

Catalonia is well known for being a bilingual region with linguistic policies that support and promote the use of Catalan. During the past few years, though, the number of languages spoken in Catalonia has increased considerably due to migratory flows and tourism. In this context, court interpreting has become an essential element when persons with limited (or null) competency in the official languages are involved in a court proceeding, either as defendants or witnesses. This article sheds light onto the study of the multilingual court based on data extracted from a corpus of transcriptions of 55 authentic trials celebrated in Barcelona where interpreting was requested. Diglossia in court proceedings is analysed at two levels: the languages used by the judiciary staff and by the allophone defendants or witnesses. The results reflect the uneven use of the two co-official languages in Catalan court settings, where Spanish is more widely used than Catalan, but also the problems that may arise from the use of a lingua franca (such as English or French) for defendants with other mother tongues. The 
results show that Spanish is clearly the dominant language during the court hearings. English and French are actually used as lingua franca in court hearing with interpreting, even though users of these languages might have different levels of competency.

Keywords: court interpreting; multilingual court; diglossia; language barriers. 


\section{Introducción}

En 2015 en España y, por extensión, en Catalunya, se aprobó la Ley Orgánica 5/2015, de 27 de abril, por la que se modificaron la Ley de Enjuiciamiento Criminal y la Ley Orgánica 6/1985, de 1 de julio, del Poder Judicial, para transponer la Directiva 2010/64/UE, de 20 de octubre de 2010, relativa al derecho a interpretación y a traducción en los procesos penales y la Directiva 2012/13/UE, de 22 de mayo de 2012, relativa al derecho a la información en los procesos penales (ver BOE del 28/4/2015). Estas modificaciones subrayan la importancia de la traducción y la interpretación como elementos de garantía procesal y de cumplimiento de otros derechos, como pueden ser el derecho a ser informados de la acusación, el derecho a un proceso público con todas las garantías y el derecho a la defensa, recogidos en el artículo 24 de la Constitución española.

Aunque estos cambios son recientes, hace años que la interpretación judicial es una realidad en los juzgados y tribunales de Catalunya, una región con altos índices de inmigración y turismo durante las últimas décadas y, por lo tanto, con una diversidad lingüística visible en todos los ámbitos de los servicios públicos. A finales de 2016, el Grupo de Estudio de las Lenguas Amenazadas (GELA) cifraba en más de 300 las lenguas habladas en Catalunya․ Según Carme Junyent, directora de este grupo, un 12\% de los catalanes "no tiene ni el catalán ni el castellano como primera lengua”2.

Los informes del Departamento de Justicia en cuanto a traducciones e interpretaciones reflejan las necesidades de interpretación judicial en los últimos años. En 2016 se prestaron 27777 servicios de interpretación judicial, lo que supuso un 8,32\% más de servicios que el año anterior (en 2015 se hicieron 25254 servicios). Las lenguas más interpretadas en juicios en 2016 fueron el árabe, el rumano, el francés, el inglés y el urdu (en ese orden), las mismas que en los años anteriores (2013-2015).

Según este mismo informe, en la ciudad de Barcelona se prestaron 12987 servicios de interpretación, lo que equivale casi a la mitad (47,04\%) del total de servicios prestados en 2016. Lo que no registran los informes es cuál fue la lengua vehicular en esos juicios (catalán o castellano), ni cuál era la lengua materna de los acusados, víctimas o testigos que necesitaron interpretación. De hecho, como se observa en el siguiente extracto, la Directiva 2010/64/UE no obliga a que la interpretación se realice en la lengua materna del acusado:

1 Según información publicada y actualizada periódicamente en su página web: http://www.gela. cat/doku.php?id=Ilengues.

2 Entrevista publicada en http://www.ub.edu/web/ub/es/menu_eines/noticies/2017/entrevistes/ carmejunyent.html. 
(22) La interpretación y traducción en virtud de la presente Directiva deben facilitarse en la lengua materna del sospechoso o acusado o en cualquier otra lengua que entienda o hable con objeto de permitir el pleno ejercicio del derecho a la defensa, y con el objeto de salvaguardar la equidad del proceso.

En cuanto a las lenguas vehiculares, diversos informes han puesto de manifiesto que el catalán es una lengua minorizada en la justicia en Catalunya (Riart, 2016). Según el Informe de política lingüística de la Generalitat de Catalunya (2017), en el período de 2015 y 2016 el porcentaje de sentencias judiciales en catalán se estabilizó en un 8,4\%. Ante esta realidad, la Dirección General de Política Lingüística de Catalunya impulsó en 2014 una campaña para fomentar el uso de esta lengua en la justicia (juzgados, registros públicos, notarías, despachos de abogados, entre otros). En 2016, 1490 profesionales del derecho y de la justicia participaron en cursos de catalán y de lenguaje jurídico3.

Este artículo se enmarca en el contexto de multilingüismo en los juicios con intérpretes en Catalunya y pretende analizar las relaciones que se establecen entre las lenguas en estos juicios. Para ello, nos basamos en un corpus de transcripciones de juicios reales celebrados en Barcelona en los que se solicitó interpretación al inglés, al francés o al rumano. De este modo, planteamos dos preguntas que servirán de eje para la investigación. En primer lugar, y habida cuenta de que el catalán es una lengua minorizada en la justicia según los estudios anteriores, nos preguntamos si el hecho de que haya interpretación hace disminuir todavía más el uso de esta lengua en los juicios analizados. En segundo lugar, existe una segunda jerarquía de lenguas: la de los acusados o testigos para quienes se solicita interpretación. En muchas ocasiones, la interpretación no se realiza a la lengua materna de estos testigos o acusados, sino a lenguas francas como pueden ser el inglés o el francés, a pesar de que pueden existir niveles muy distintos de competencia comunicativa en estas segundas lenguas. Por lo tanto, nos preguntamos cómo es la comunicación cuando la interpretación no se hace a la lengua materna del acusado o testigo: ¿en qué medida se puede hablar de comunicación efectiva que realmente cumpla el derecho a la información que pretende reforzar la Directiva 2010/64/UE?

\section{Bilingüismo y multilingůismo en los servicios públicos en Catalunya}

El artículo 15 de la Declaración universal de derechos lingüísticos incluye disposiciones relativas al uso de la lengua propia en la administración pública y los órganos oficiales, y reconoce explícitamente que "toda comunidad lingüística tiene derecho a que las actuaciones judiciales y administrativas, los documentos públicos y privados y los asientos en registros públicos realizados en la lengua propia del territorio sean válidos y eficaces y nadie pueda alegar el desconocimiento".

3 Véase http://lengua.gencat.cat/web/.content/documents/informepl/arxius/IPL2016_web_v1.pdf. 
En España, la cooficialidad de las lenguas regionales en sus respectivos territorios se establece en el artículo 3.2 de la Constitución de 1978:

Artículo 3.

3.1. El castellano es la lengua española oficial del Estado. Todos los españoles tienen el deber de conocerla y el derecho a usarla.

3.2. Las demás lenguas españolas serán también oficiales en las respectivas Comunidades Autónomas de acuerdo con sus Estatutos.

3.3. La riqueza de las distintas modalidades lingüísticas de España es un patrimonio cultural que será objeto de especial respeto y protección.

En este contexto, la situación sociolingüística en Catalunya es compleja, porque, como suele pasar en regiones bilingües, la elección de una lengua u otra por parte de los hablantes está determinada por factores diversos, como pueden ser los que propone Fishman (2000): el sentimiento de pertenencia al grupo, la situación o el tema de la conversación. Tanto el catalán como el castellano son lenguas cooficiales que deberían ser aceptadas en todos los servicios públicos —según la clasificación de Meylaerts (2011), podríamos decir que en Catalunya existe un multilingüismo institucional completo que conlleva traducciones multidireccionales obligatorias para todos-; en la práctica las diferencias en el uso son bastante claras.

Mientras que la inmersión lingüística en la escuela catalana ha sido un éxito (cf. Strubell, 1996), en la justicia, como ya se ha mencionado, la situación de diglosia es evidente. Un informe de Plataforma per la Llengua (2017) analiza los motivos para el uso tan reducido del catalán en el ámbito de la justicia. De entre once motivos, la procedencia de otras comunidades autónomas del personal de justicia es quizás uno de los mayores obstáculos para un uso más extendido del catalán en este ámbito. Las leyes que regulan el acceso a la administración de jueces, magistrados, fiscales o procuradores (véase, por ejemplo, la Ley 6/1985 del Poder Judicial y el Reglamento 2/2011 de la carrera judicial) solo incluyen los conocimientos de catalán como mérito, no como requisito. Como consecuencia, no todos los operadores judiciales que trabajan en Catalunya tienen conocimientos de catalán. Otros de los motivos indicados en el informe de Plataforma per la Llengua son la ausencia de materiales de trabajo en catalán (por ejemplo, manuales, códigos anotados o legislación), la lengua de redacción de los procedimientos judiciales (por defecto, en castellano) o el hecho de que el castellano sea la única lengua de la justicia en las instituciones centrales y superiores (Ley orgánica 6/1985 del Poder Judicial).

En cualquier caso, las diferencias en el uso del catalán coinciden con la descripción que propone Diaz Fouces para las lenguas minorizadas:

4 Uno de los ejemplos más extremos que demuestran que este multilingüismo institucional no siempre es real es el caso recopilado por Òscar-Adrià Ibáñez Ferreté y Eloi Torrents Vivó (2016) en el que una jueza amenazó a una testigo con suspender el juicio si hablaba en catalán. 
As far as allocation of use is concerned (...), the interference of the dominant languages is very strong. The members of the minoritized linguistic community have to abide by patterns of bilingual behaviour, whereas the members of the dominant community can use their own language in all or most circumstances within the same territory (2005: 96).

Desde los planes de inmigración también se ha intentado promover y normalizar el uso del catalán entre la población de origen extranjero. De hecho, el Plan de Ciudadanía e Inmigración 2005-08 (Generalitat de Catalunya, 2006) incluía el objetivo explícito de "la defensa de la lengua y de la construcción de una identidad catalana más fuerte, rica y diversa” (p. 40). Según este plan, todos los ciudadanos que residen en Catalunya, independientemente de su lugar de origen, deberían ser capaces de comunicarse en catalán con la administración pública. En este sentido, Climent Ferrando (2016) sostiene que el análisis de los discursos sobre lengua e inmigración en Catalunya "revela la construcción de un marco estratégico que positiviza discursivamente la inmigración con el objetivo político de captar la empatía de los inmigrantes e incorporarlos al proyecto de construcción nacional”.

En el caso de las barreras lingüísticas, la promoción de la lengua propia puede chocar con las necesidades de comunicación en servicios básicos como la salud o la educación. García-Beyaert (2015: 210), después de analizar la situación de Ontario, sugiere que, en aras de establecer medidas efectivas para superar las barreras comunicativas que suponen las diferencias lingüísticas, la sociedad debería dejar atrás la dimensión identitaria de las lenguas. En otras palabras, facilitar la comunicación debería prevalecer por encima de las políticas lingüísticas.

Según la terminología propuesta por Ozolins (2010), se puede afirmar que Catalunya carece de "políticas lingüísticas transversales" en cuanto a la gestión del multilingüismo y de las barreras lingüísticas en los servicios públicos, lo que la ha llevado a adoptar "políticas específicas para cada ámbito" en concreto. Así, en cuanto al sistema judicial, la interpretación en juicios es competencia del Departamento de Justicia, que es quien convoca las licitaciones públicas para la provisión de la interpretación en tribunales y juzgados, lo que se conoce como el "modelo de contratas" (Blasco Mayor y Del Pozo Triviño, 2015). Las empresas adjudicatarias suelen ser siempre las mismas, por el hecho de ofrecer una mayor cobertura horaria y mayor cantidad de lenguas, además del presupuesto más ajustado. Este modelo, que fue adoptado como reacción al gran aumento en la necesidad de intérpretes judiciales que se produjo a finales de los años 1990, ha sido objeto de duras críticas. Blasco Mayor y Del Pozo Triviño (2015: 16) explican que las empresas adjudicatarias "son las encargadas de escoger a los traductores e intérpretes, de establecer los requisitos para su contratación (mínimos en la mayoría de los casos), así como de determinar sus condiciones de trabajo: horario, remuneración, y demás".

La remuneración, de hecho, puede llegar a ser tan baja (10-15 € por hora según Gascón Nasarre, 2011) que ejerce un efecto disuasivo entre los profesionales formados que se podrían 
sentir atraídos por este ámbito laboral. La Administración de Justicia no establece ningún control ni proceso de selección de estos profesionales, responsabilidad que delega en las empresas adjudicatarias. De este modo, a menudo se contrata a personas con competencia comunicativa insuficiente para las lenguas que tendrán que interpretar. La baja remuneración tampoco contribuye a que estas personas que realizan la labor de la interpretación se interesen por cursos de especialización en este ámbito.

\section{Descripción del estudio: el proyecto TIPp}

Aunque el tema de la interpretación judicial ha generado mucho interés a nivel internacional, la investigación en Catalunya ha sido relativamente escasa hasta el proyecto TIPp (20152017) 5 . De entre las pocas excepciones anteriores a este proyecto, cabe mencionar la tesis de Onos (2014), que explora el caso de la interpretación judicial del rumano en Barcelona. Sus resultados ponen de manifiesto la preparación insuficiente de los intérpretes y la falta de recursos específicos para la interpretación del rumano, entre otros aspectos.

La necesidad de estudios que tuvieran en cuenta una perspectiva más global de la interpretación judicial y que se basaran en una recogida de datos más sistemática fue lo que impulsó, en gran medida, el proyecto TIPp, “Traducción e interpretación en los procesos penales”, del grupo MIRAS. Este proyecto se ha basado en la creación, anotación y análisis de un corpus de transcripciones de juicios reales para los que se solicitó interpretación (Orozco Jutorán, 2017; Arumí y Vargas-Urpi, 2018).

La creación de este corpus ha sido posible porque el grupo de investigación MIRAS obtuvo autorización para acceder a las grabaciones de las vistas con interpretación de diez Juzgados de lo Penal de Barcelona para el periodo 2010-2015. El corpus estudiado está compuesto por las transcripciones de las vistas celebradas durante la primera mitad de 2015 (enero-junio) en las que hubo interpretación entre las lenguas vehiculares del juicio (castellano o catalán) y el inglés, francés o rumano. La tabla 1 muestra los detalles de este corpus (véase la tabla en la página siguiente).

La tabla indica explícitamente el número de juicios en los que la interpretación se solicitó para la persona acusada (y no para un testigo, por ejemplo), porque estos son los casos en los que la interpretación debería contribuir a garantizar los derechos del acusado según las Directivas 2010/64/UE y 2012/13/UE del Parlamento Europeo.

Paralelamente a la construcción del corpus, se creó una hoja de cálculo con los metadatos de los juicios, donde se anotaron las características de cada juicio, como pueden ser

5 Para más información, véase http://pagines.uab.cat/tipp/. 


\section{TABLA 1}

Número de vistas del corpus y duración en minutos

\begin{tabular}{lccc}
$\begin{array}{l}\text { LENGUA DE } \\
\text { INTERPRETACIÓN } \\
\text { SOLICITADA }\end{array}$ & VISTAS & $\begin{array}{c}\text { EL USUARIO DE LA } \\
\text { INTERPRETACIÓN } \\
\text { ES LA PERSONA } \\
\text { ACUSADA }\end{array}$ & $\begin{array}{c}\text { DURACIÓN } \\
\text { TOTAL } \\
\text { (MINUTOS) }\end{array}$ \\
\hline Inglés & 19 & 18 & 371 \\
\hline Francés & 9 & 7 & 190 \\
\hline Rumano & 27 & 26 & 555 \\
\hline Total & 55 & 51 & 1116 \\
\hline
\end{tabular}

el tipo de delito juzgado, las lenguas vehiculares (castellano, catalán, o ambas) o si había evidencias de que el acusado o testigo estuviera empleando una lengua distinta a la de la interpretación (es decir, una segunda lengua como lengua franca).

Los objetivos principales del proyecto están orientados al análisis de la calidad de la interpretación en los juicios (detección de errores, análisis de las técnicas de traducción, estrategias de interpretación, uso de la primera o tercera persona, entre otros aspectos) con el fin último de poder aportar recomendaciones que contribuyan a la mejora de la interpretación judicial, tanto para los intérpretes como para los operadores judiciales (jueces, magistrados, abogados, ministerio fiscal, auxilio judicial), así como recursos específicos para la traducción de la terminología más frecuente en las combinaciones lingüísticas estudiadas ${ }^{6}$. Para este artículo, sin embargo, aprovechamos la existencia del corpus de transcripciones y de las tablas de metadatos para analizar los juicios desde la sociología del lenguaje, una perspectiva que prácticamente no se ha empleado en los estudios sobre interpretación judicial. Concretamente, el artículo pretende explicar las relaciones jerárquicas que se establecen entre las lenguas de los participantes, combinando datos cuantitativos extraídos de las tablas de metadatos, con datos de tipo cualitativo obtenidos del análisis de los fragmentos en los que se produce alternancia de código (code-switching).

\section{Uso del catalán en el corpus de juicios estudiados}

Según la información de la hoja de cálculo con los metadatos de los juicios, la tabla 2 muestra las lenguas vehiculares que se emplearon en el corpus estudiado.

6 Véase http://interpretacionenprocesospenales.es/web/index.php. 


\section{TABLA 2}

Lenguas vehiculares de los juicios

\section{LENGUA DE \\ INTERPRETACIÓN \\ SOLICITADA}

\section{N. ${ }^{\circ}$ DE VISTAS}

\begin{tabular}{ccc}
\multicolumn{3}{c}{ LENGUA VEHICULAR DEL JUICIO } \\
\hline SOLO & SOLO & CASTELLANO \\
CASTELLANO & CATALÁN & Y CATALÁN
\end{tabular}

\begin{tabular}{lcccc} 
Inglés & 19 & 18 & 0 & 1 \\
\hline Francés & 9 & 8 & 0 & 1 \\
\hline Rumano & 27 & 19 & 0 & 8 \\
\hline Total & 55 & 45 & 0 & 10 \\
\hline
\end{tabular}

Como se puede observar, no hay ninguna vista del corpus que se celebre íntegramente en catalán. El catalán se utiliza en algún momento en 10 vistas, sin llegar a ser la lengua mayoritaria en ningún caso. Además, cabe señalar que la lengua de la interpretación de lo que dicen los acusados o testigos siempre es el castellano, independientemente de la lengua empleada por los operadores judiciales en la vista en cuestión.

La tabla 3 desglosa la duración de los fragmentos en catalán en las 10 vistas en las que se utiliza esta lengua en algún momento.

\section{TABLA 3}

Uso del catalán en los juicios en los que se alternan las dos lenguas vehiculares

\begin{tabular}{|c|c|c|c|c|}
\hline $\begin{array}{l}\text { LENGUA DE } \\
\text { INTERPRETACIÓN } \\
\text { SOLICITADA }\end{array}$ & $\begin{array}{l}\text { REF. } \\
\text { VISTA }\end{array}$ & $\begin{array}{l}\text { DURACIÓN TOTAL } \\
\text { DE LA GRABACIÓN } \\
\text { (MINUTOS) }\end{array}$ & $\begin{array}{l}\text { DURACIÓN } \\
\text { DE LA PARTE } \\
\text { EN CATALÁN }\end{array}$ & $\begin{array}{l}\text { \% DE CATALÁN } \\
\text { USADO EN } \\
\text { LA VISTA }\end{array}$ \\
\hline \multirow{8}{*}{ Rumano } & 84 & 22 & 8:39 & $39 \%$ \\
\hline & 365 & 12 & $2: 04$ & $16 \%$ \\
\hline & 336 & 13 & $0: 16$ & $2 \%$ \\
\hline & 339 & 12 & 3:39 & $30 \%$ \\
\hline & 415 & 16 & $0: 55$ & $6 \%$ \\
\hline & 18 & 23 & $0: 57$ & $4 \%$ \\
\hline & 393 & 29 & 10:18 & $35 \%$ \\
\hline & 81 & 60 & 1:52 & $3 \%$ \\
\hline Francés & 130 & 20 & $1: 45$ & $9 \%$ \\
\hline Inglés & 254 & 25 & 1:39 & $6 \%$ \\
\hline Total subcorpus & 10 vistas & 232 & 32 minutos & $14 \%$ \\
\hline
\end{tabular}


Como se puede observar, de los 232 minutos de duración de las 10 vistas, el uso del catalán solamente ocupa 32 minutos, es decir un $14 \%$ del subcorpus, lo que equivale a un 2,82\% de la duración del corpus total de 55 vistas (1116 minutos). Comparativamente, el uso del catalán parece ser mayor en las vistas con interpretación al rumano, llegando al 39\% de la duración total de la vista en uno de los casos (ref. 84).

El análisis cualitativo de los fragmentos en los que se emplea catalán aporta también información relevante. Por ejemplo, permite observar en qué momentos se utiliza el catalán en estas vistas, a saber:

- En 5 vistas es el juez quien utiliza el catalán para presentar a alguno de los testigos (sobre todo en el caso de los Mossos d’Esquadra, la policía autonómica en Catalunya). En todos estos casos, después de la presentación en catalán por parte del juez, el fiscal que continúa con el interrogatorio lo hace en castellano.

- En 4 vistas, el uso del catalán se debe a que el letrado de la defensa habla en esta lengua y, por este motivo, algunos fragmentos de la vista (por ejemplo, el interrogatorio por parte de este letrado o su informe) son en catalán. Estos casos se corresponden con los juicios con una mayor proporción de catalán hablado (39\%, 35\%, 30\% y 16\% de las vistas con interpretación al rumano).

- En 1 vista es el fiscal quien lee una minuta policial en catalán y el interrogatorio subsiguiente continúa unos minutos en esta lengua.

El análisis cualitativo también permite observar cómo se produce la alternancia de código en estas vistas en las que, en teoría, el intérprete debería estar interpretando todo al acusado. Solo en dos casos se pregunta al intérprete si entiende el catalán, como veremos a continuación. En el resto de los casos, o bien hay algún participante que habla en catalán de forma habitual (el letrado de la defensa en 4 vistas), o bien un mismo participante produce una alternancia de código, como se observa en el extracto 1.

\section{Extracto 1.}

Letrado de la acusación particular: Vale. Ninguna pregunta más señoría. Gracias.

Juez: La defensa de l'altre acusat té alguna pregunta?

[¿La defensa del otro acusado tiene más preguntas?]

Letrado de la defensa: Sí, amb la vènia. Eh, ¿el señor Ion salió corriendo y usted vio que se marchó del lugar?

[Sí, con la venia]...

7 Utilizamos el verbo "debería” porque uno de los resultados más relevantes del Proyecto TIPp es que solo se interpreta (en voz alta o susurro) un 46\% de las vistas (Arumí y otros, 2017). 
En el extracto 1, el letrado de la defensa produce una alternancia de código (catalán-castellano) en un mismo turno, debida al cambio de interlocutor. Contesta en catalán al juez, porque este se ha dirigido a él en catalán, pero luego continúa con el interrogatorio en castellano.

Los extractos siguientes muestran los dos casos en los que se pregunta al intérprete si entiende el catalán antes de seguir con esta lengua. Ambos casos pertenecen al subcorpus de vistas con interpretación al rumano. En el primer caso (extracto 2), el testigo policial parece dudar sobre qué lengua usar, el juez le anima a hablar en catalán y, a continuación, pregunta al intérprete si entiende esta lengua. Paradójicamente, aunque el intérprete dice que sí entiende el catalán, el interrogatorio posterior sigue en castellano.

\section{Extracto 2.}

Juez: Hola, bon dia. ¿Me puede decir su número TIP de mosso d'esquadra? [Hola, buenos días.] (...)

Testigo policial: Sí, señoría. Cero... Zero...

(...) [Cero...]

J: Si vol parlar en català, pot parlar en català.

[Si quiere hablar en catalán, puede hablar en catalán.]

TP: Com vulgui.

[Como quiera.]

J: Eeh, l'intèrpret entén el català... de català?

[Eeh, ¿el intérprete entiende el catalán... de catalán?]

Intérprete: Sí, perfectamente.

J: Si quiere hablar catalán, pues puede hablar catalán.

TP: Molt bé.

[Muy bien.]

J: Eh, pues tiene la palabra el ministerio fiscal.

Fiscal: Sí, con la venia señoría. ¿Ratifica la, la, la minuta policial?

TP: Sí, afirmativo, la ratifico.

En el extracto 3 observamos, de nuevo, la inseguridad en el uso del catalán ante los fragmentos que deberían ser interpretados. Igual que en el caso anterior, en primer lugar, se produce una negociación de la lengua con el testigo que va a ser interrogado (en este caso, la víctima del delito) y luego se consulta a la intérprete por su comprensión del catalán.

\section{Extracto 3.}

Letrado de la defensa: Ninguna pregunta, señoría.

Juez: Val, que passi el primer testimoni. (...) Hola, bon dia.

[Vale, que pase el primer testigo. (...) Hola, buenos días.]

Testigo (víctima) 1: Hola, bon dia.

[Hola, buenos días]. 


\section{J: Senyor José Fernández..}

[Señor José Fernández.]

TV1: Sí.

J: Eh, mej---, millor en castellà o en català? A mi m'és igual.

[Eh, mej --- ¿mejor en castellano o en catalán? A mí me da igual.]

TV1: Bueno, me es igual.

J: Bueno, eh, primer li explicaré els seus drets i obligacions en català i després, si no l'importa, parlarem en castellà perquè més o menys ens pugui entendre la senyora intèrpret. Porque, ¿usted entiende el catalán?... No, ¿iverdad?

[Bueno, eh, primero le explicaré sus derechos y obligaciones en catalán y después, si no le importa, hablaremos en castellano para que más o menos nos pueda entender la señora intérprete. (...)]

\section{Intérprete: Sí.}

J: Bueno. Bueno, cadascú que parli com vulgui. Va a declarar com testimoni, jura o promet dir la veritat?

[Bueno. Bueno, que cada uno hable como quiera. Va a declarar como testigo, ¿jura o promete decir la verdad?]

TV1: Sí.

J: Li tinc d'advertir que si no digués la veritat, podria cometre un delicte que el Codi Penal castiga amb pena de presó. D’acord? Primer li preguntarà la senyora fiscal. Senyora fiscal? [Le tengo que advertir que, si no dijera la verdad, podría cometer un delito que el Código Penal castiga con pena de prisión. ¿De acuerdo? Primero le preguntará la señora fiscal. ¿Señora fiscal?]

F: Con la venia, señoría.

(A partir de aquí, el interrogatorio sigue en castellano).

Este ejemplo es interesante por varios aspectos. En primer lugar, se observa la selección que hace el juez de la información que debería ser interpretada ("Bueno, eh, primero le explicaré sus derechos y obligaciones en catalán y después, si no le importa, hablaremos en castellano para que más o menos nos pueda entender la señora intérprete"), lo que le lleva a recomendar el uso del castellano para facilitar la labor a la intérprete. En segundo lugar, la pregunta, formulada en negativo (“¿Usted entiende el catalán?... No, ¿verdad?”), demuestra la poca información que se tiene de los intérpretes y revela, también, un posible prejuicio hacia estos profesionales. La conclusión de "que cada uno hable como quiera" refleja la aceptación del bilingüismo durante el juicio por parte del juez.

Finalmente, cabe señalar que en 6 de las 10 vistas en las que se utiliza catalán, el uso de esta lengua no es impedimento para que haya interpretación mediante susurro (parcial, en la mayoría de casos) de lo que se está diciendo. El susurro de información en catalán se suele producir en los juicios en los que los fragmentos en esta lengua son más largos (ref. 84, 365, 339, 415, 393, 130 de la tabla 3). 


\section{El uso de segundas lenguas en el corpus estudiado}

En ocasiones, debido a la falta de intérpretes de ciertas lenguas maternas (por ejemplo, en el caso de lenguas africanas), se recurre a lenguas francas como pueden ser el inglés o el francés, para las que la disponibilidad de intérpretes suele ser mayor. Díaz explica que

cuando una persona africana ha de realizar declaraciones o solicitudes, con frecuencia se le pide que sea en la lengua oficial de su país, que suele ser el francés, el inglés, el portugués u otra lengua de la metrópoli colonizadora. Si es solicitante de asilo, tendrá derecho a un intérprete. Sin embargo, un representante de la CEAR (Comisión Española de Ayuda al Refugiado) afirma que a veces "se fuerza a los extranjeros a expresarse en una lengua que, aunque sea oficial en su país de origen, no controlan" (Gara, 2001). Al encontrarse en una situación psicológicamente delicada como es la llegada a un país desconocido en busca de refugio político, le será más difícil hablar con soltura esta lengua (2004: 16).

Cabe señalar que las lenguas maternas de los acusados no constan en ningún registro: ni en los informes que publica el Departamento de Justicia ni en las listas de uso interno que nos facilitaron los gestores del servicio de interpretación. En dichas listas, solo aparecen las lenguas para las que se solicita interpretación, siendo el francés y el inglés la tercera y la cuarta lenguas con más solicitudes de interpretación en los últimos años, como se ha visto en la introducción. Por este motivo, uno de los datos que decidimos registrar en las tablas de metadatos era si en los juicios con interpretación de inglés o francés podíamos tener evidencias de que estas lenguas se estuvieran utilizando como lenguas francas (para acusados o testigos con otras lenguas maternas). Los técnicos encargados de ver los juicios y transcribirlos anotaron aquellos casos en los que se podía saber, claramente, que se estaba utilizando el inglés o el francés como lengua franca, los casos en los que se veía con certeza que estas eran las lenguas maternas de los declarantes y los casos en los que no existían suficientes evidencias para determinar esta cuestión. La tabla 4 muestra los resultados obtenidos.

\section{TABLA 4}

Lenguas maternas del acusado/testigo para quien se solicita la interpretación

\begin{tabular}{lcccc}
$\begin{array}{l}\text { LENGUA DE } \\
\text { INTERPRETACIÓN } \\
\text { SOLICITADA }\end{array}$ & $\begin{array}{c}\text { NÜMERO } \\
\text { DE VISTAS }\end{array}$ & $\begin{array}{c}\text { LENGUA MATERNA } \\
\text { = LENGUA } \\
\text { SOLICITADA }\end{array}$ & $\begin{array}{c}\text { LENGUA MATERNA } \\
\text { fLENGUA } \\
\text { SOLICITADA }\end{array}$ & $\begin{array}{c}\text { EVIDENCIAS } \\
\text { INSUFICIENTES }\end{array}$ \\
\hline Inglés & 19 & 1 & 17 & 1 \\
\hline Francés & 9 & 8 & 2 & 5 \\
\hline Total & 28 & 9 & 19 & 6 \\
\hline
\end{tabular}

Como se observa en la tabla 7, a partir de las grabaciones del corpus se puede afirmar que en 19 vistas (17 del inglés y 2 del francés) los acusados o testigos se expresaban en una segunda 
lengua y no en su lengua materna. Se hace bastante evidente el uso del inglés como lengua franca en los juicios que se celebran con interpretación a esta lengua. La imposibilidad de arrojar más luz sobre este tema pone de manifiesto la "invisibilidad” de las lenguas maternas de las personas para las que se solicita la interpretación.

En cuanto a posibles problemas derivados del uso de lenguas francas, en primer lugar cabe señalar dificultades en la comprensión de lo que se está interpretando al inglés o al francés. Por ejemplo, en el extracto 4, la acusada parece no entender bien qué se le está preguntando.

\section{Extracto 4.}

Fiscal: Pues, ¿se encontraban juntas cuando llegó la policía?

Intérprete: But were you together when police arrived?

Acusada 4: Hmm?

I: Were you together when police arrived?

A4: I don't understand.

I: Were you together when police arrived?

A4: We were three.

I: Estaban tres, antes.

A4: So they took us to Comisaría, and later, later, I surprised, these two girls came back again (...)

La acusada parece no entender una pregunta que no requiere un nivel de competencia comunicativa alto, en la que no aparece terminología específica ni tampoco una estructura lingüística especialmente compleja. La intérprete repite hasta tres veces la misma pregunta y la respuesta que recibe tampoco se corresponde con la pregunta realizada.

El extracto anterior también refleja dificultades en la expresión (por ejemplo, dice "I surprised” cuando, supuestamente, parece que querría decir “I was surprised”). En otros fragmentos esta expresión que refleja una baja competencia comunicativa en la lengua franca puede incluso llevar a ambigüedades, como se ve en el extracto 5.

\section{Extracto 5.}

Fiscal: Y el motivo de que estuviera Heba en el domicilio, ¿por qué es?

Intérprete: So, why was Heba in the flat?

Acusado: Hmmm... Omarbring her home.

Intérprete: Que... le(.) Omar la había traído a:... al... piso...

Aunque por el contexto parece que el acusado quería hablar en pasado (brought, had brought), también podría darse el caso de que quisiera utilizar un presente simple (brings) para referirse a un acto que se repite habitualmente. De este modo, se trata de una respuesta ambigua originada, probablemente, por el hecho de estar hablando una segunda lengua. 


\section{Discusión y conclusiones}

Con este artículo, a pesar de partir de un corpus limitado, se ha intentado aplicar la perspectiva de la sociología del lenguaje a un corpus de juicios con interpretación celebrados en Barcelona. Probablemente, el resultado más llamativo es la situación de diglosia en dos niveles distintos: por un lado, la diglosia entre las lenguas vehiculares del juicio y, por el otro, la diglosia entre las lenguas usadas por los acusados o testigos alófonos.

De este modo, en cuanto a las lenguas vehiculares, el análisis de los 55 juicios del corpus revela datos incluso inferiores al porcentaje de sentencias en catalán: se habla en catalán en tan solo un 2,8\% del corpus total de vistas, mientras que los informes de la Administración indican que las sentencias en esta lengua llegan al 8,4\%. Los datos corroboran, de este modo, la minorización que sufre el catalán en el ámbito de la justicia.

El mayor uso del castellano en los juicios está motivado por muchos factores, como se ha visto en la segunda sección. En el caso de los juicios de nuestro corpus, se podría argüir que el hecho de tener que ser interpretados disuade a los participantes del uso de una lengua considerada minoritaria como es el catalán. En los dos casos en los que esta duda se manifiesta verbalmente, como hemos visto, los intérpretes afirman entender catalán. Sin embargo, sería necesario tener más datos sobre su nivel de competencia en esta lengua, pues para poder entender e interpretar un juicio, no bastaría con "entender catalán”, deberían tener un nivel C1/C2 del Marco Común Europeo de Referencia. Por otro lado, el hecho de que en 6 de las 10 vistas en las que se habla catalán también se interpreten, aunque sea parcialmente, los fragmentos en los que se habla esta lengua, también pone de manifiesto que al menos parte de los intérpretes judiciales la entiende y es capaz de interpretarla. En otras palabras: el uso del catalán por parte de los operadores judiciales no implica un menor empleo de la interpretación susurrada por parte de los intérpretes, que se mantiene en proporciones parecidas a las del corpus global. Para tener mayor contexto, cabe señalar que, en el corpus global, raramente se interpreta mediante susurro la vista entera: esto solo ocurre en un $9,8 \%$ de las vistas. De media, un 54\% de la vista no se interpreta.

En el corpus analizado no se utiliza el catalán al inicio de la vista o en el informe final. En cambio, suele aparecer sobre todo en la parte del interrogatorio de testigos, como hemos visto en los ejemplos en los que el juez pasa al catalán para dirigirse a las personas que serán interrogadas. La casuística analizada parece reflejar dos fenómenos en cuanto al uso del catalán: por un lado, habría los operadores judiciales (letrados de la defensa) que, por norma, se expresan en catalán, y, por el otro, operadores judiciales (jueces) que producen una alternancia de código del castellano al catalán en función del interlocutor a quien se dirigen.

La alternancia de código entre catalán y castellano sucede, en general, de manera espontánea - como suele pasar en la vida cotidiana-, sin entorpecer la comunicación fluida, al menos en el corpus estudiado. En los casos de alternancia de código, se impone la 
jerarquía diglósica por encima de la jerarquía institucional; es decir, aunque en ocasiones el juez es quien habla en catalán o incluso invita a hablar en catalán (como hemos visto en el extracto 3), los demás operadores judiciales o participantes en el juicio acaban expresándose en castellano.

El segundo nivel de diglosia es el que corresponde a las lenguas maternas de los acusados con respecto de las lenguas francas como el inglés o el francés, que son las que se utilizan para la interpretación. De este modo, las lenguas maternas acaban siendo lenguas minorizadas e invisibilizadas, porque no constan en ningún registro, mientras que las lenguas francas adoptan el rol de lenguas "oficiales" para la comunicación mediante intérpretes. En este aspecto, habría que matizar un detalle: en algunas comunidades muy reducidas, es posible que fuera el mismo acusado quien pidiera no tener un intérprete de su propia lengua materna para poder preservar su anonimato y la confidencialidad del juicio.

En relación con esta última cuestión, posiblemente la mayor limitación del presente estudio es el hecho de haberse basado en grabaciones existentes, lo que implica también la imposibilidad de conseguir más datos de los participantes en las grabaciones. De haber sido posible, habría sido interesante recopilar información sobre el uso de las lenguas por ejemplo, mediante entrevistas o cuestionarios a todos los participantes en las vistas analizadas (jueces, fiscales, acusados, testigos, letrados, intérpretes, entre otros) - con el fin de poder llegar a conclusiones más firmes sobre las motivaciones para la elección de la lengua empleada. En cualquier caso, los datos son igualmente relevantes porque se han extraído de un corpus auténtico y espontáneo y, por lo tanto, reflejan la realidad de los juicios multilingües estudiados.

Cabe recordar, finalmente, que la interpretación responde al derecho de información por parte de los acusados y que, por lo tanto, cuando no se interpreta durante un juicio o cuando se hace en una lengua en la que los acusados cuentan con una competencia limitada, estos se encuentran en situación de indefensión. En este sentido, quizás sería bueno, como mínimo, empezar a visibilizar las lenguas maternas de los acusados cuando estas difieren de la lengua de la interpretación.

Por cuestiones de enfoque y espacio, no se discute, en este artículo, la calidad cuestionable de la interpretación judicial en Catalunya, aunque sin duda es el gran problema detectado y descrito a raíz del proyecto TIPp (véase, por ejemplo, Arumí y otros, 2017).

\section{Bibliografía citada}

ARUmi, Marta, y Mireia VARGAS-URPI, 2018: "Annotation of interpreters' conversation management problems and strategies in a corpus of criminal trials in Spain: the case of non-renditions", Translation and Interpreting Studies 13 (3), 421-441. 
Arumí, Marta, Carmen Bestué Salinas, Anna Gil-Bardají, Mariana Orozco Jutorán, Mireia Vargas-Urpi y Francisco VIGIER, 2017: La qualitat de la traducció com a factor de garantia del procés penal: desenvolupament de recursos per a intèrprets judicials (el projecte TIPp). Informe de prensa, Universitat Autònoma de Barcelona [https://ddd.uab.cat/record/176075, fecha de consulta: 3 de febrero de 2018].

Blasco Mayor, María Jesús, y Maribel Del Pozo Triviño, 2015: "La interpretación judicial en España en un momento de cambio", MonTI: Monografías de traducción e interpretación 7, 9-40

Climent-FerRando, Vicent, 2016: The European politics on language for immigrant integration: a multilevel comparative perspective. Tesis doctoral, Universitat Pompeu Fabra.

Departament de Justicia, 2017: Dades estadístiques d'interpretacions i traduccions judicials 2016, Generalitat de Catalunya [http://administraciojusticia.gencat.cat/web/.content/home/seccions_tematiques/servei_de_traduccions_i_interpretacions/informe_interpretacions_traduccions_2016.pdf, fecha de consulta: 3 de febrero de 2018].

Díaz, Beatriz, 2004: Lenguas y comunicación en la emigración, Bilbao: Traficantes de Sueños; Likiniano elkartea.

Diaz Fouces, Oscar, 2005: "Translation Policy for Minority Languages in the European Union. Globalisation and Resistance” en Albert Branchadell y Lovell M. West (eds.): Less Translated Languages, Amsterdam y Philadelphia: John Benjamins: 95-104,

Directiva 2012/13/UE del Parlamento Europeo y del Consejo, de 22 de mayo de 2012, relativa al derecho a la información en los procesos penales [https://www.boe.es/doue/2012/142/ L00001-00010.pdf, fecha de consulta: 3 de febrero de 2018].

Directiva 2010/64/UE del Parlamento Europeo y del Consejo, de 20 de octubre de 2010, relativa al derecho a interpretación y a traducción en los procesos penales [https://www.boe.es/ doue/2010/280/L00001-00007.pdf, fecha de consulta: 3 de febrero de 2018].

Fishman, Joshua, 2000 [1965]: “Who speaks what language to whom and when?” en Li Wel (ed.): The Bilingualism Reader, London y New York: Routledge, 89-106.

Garcia-Beyaert, Sofía, 2015: Cross-linguistic communication and public policy: the institutionalization of community interpreting. Tesis doctoral, Universitat Autònoma de Barcelona.

GaSCón NASARRE, Fernando, 2011: “Una breve radiografía de la interpretación judicial en España”, La Linterna del Traductor 6, 31-40. 
Generalitat de Catalunya, 2006: Pla de ciutadania i immigració 2005-2008 [http://ibdigital.uib. es/greenstone/collect/portal_social/archives/gencato1/42.dir/gencat0142.pdf, fecha de consulta: 3 de febrero de 2018].

Generalitat de Catalunya, 2017: Informe de política lingüística [http://llengua.gencat.cat/ web/.content/documents/informepl/arxius/IPL2016_web_v1.pdf, fecha de consulta: 3 de febrero de 2018].

Ibẫez Ferreté Òscar-Adrià, y Eloi Torrents Vivó, 2016: "Si me hablas en catalán se suspende el juicio" Els drets lingüístics en via morta, Plataforma per la Llengua [https://www.plataformaIlengua.cat/media/upload/pdf/2016-03-10-discriminacions-linguistiques_1457689364.pdf, fecha de consulta: 3 de febrero de 2018].

MeYlaERTS, Reine, 2011: "Translational Justice in a Multilingual World: An Overview of Translational Regimes", Meta: Journal des Traducteurs / Meta: Translators' Journal 56 (4), 743-757.

Onos, Liudmila, 2014: La interpretación en el ámbito judicial: el caso del rumano en los tribunales de Barcelona. Tesis doctoral, Universidad Autónoma de Barcelona.

Orozco-Jutorán, Mariana, 2017: "Anotación textual de un corpus multilingüe de interpretación judicial a partir de grabaciones de procesos penales reales", Revista de Llengua i Dret, Journal of Language and Law 68, 33-56.

Ozolins, Uldis, 2010: "Factors that determine the provision of Public Service Interpreting: comparative perspectives on government motivation and language service implementation", JoSTrans: The Journal of Specialised Translation 14, 194-215.

Plataforma per la Llengua, 2017. Quins són els obstacles que impedeixen l'ús del català a la justícia? [https://www.plataforma-Ilengua.cat/media/upload/pdf/informe-de-la-situacio-del-catala-a-la-justicia_cor_1490877172.pdf, fecha de consulta: 24 de septiembre de 2018].

RIART, Montse, 2016: “El català, Ilengua minoritària a la justicia”, Ara.cat, 17 de abril [http:// www.ara.cat/dossier/catala-llengua-minoritaria-justicia_0_1560444005.html, fecha de consulta: 3 de febrero de 2018].

Strubell, Miquel, 1996: "Language Planning and Bilingual Education in Catalonia”, Journal of Multilingual and Multicultural Development 17 (2-4), 261-275. 